\title{
Mastitis granulomatosa idiopática: 10 años de experiencia en el Centro de Imagenología del Hospital Clínico de la Universidad de Chile
}

\author{
Tamara Ramírez $P^{1,2}$, Alejandra López $P^{3}$, Patricia Arancibia $H^{3}{ }^{3}$, Carla Sáez T. ${ }^{3}$, \\ Lorena Díaz C. ${ }^{3}$, Teresa Taub E. ${ }^{3}$, Leonor Moyano S. ${ }^{4}$
}

\footnotetext{
${ }^{1}$ Residente, Programa de Radiología, Hospital Clínico de la Universidad de Chile. ${ }^{2}$ Magister en Ciencias Biomédicas, Mención Morfología. Facultad de Medicina Universidad de Chile. ${ }^{3}$ Radiólogo, Centro de Imagenología, Unidad de Imágenes Mamarias, Hospital Clínico de la Universidad de Chile. ${ }^{4}$ Médico Anatomopatólogo, Servicio de Anatomía Patológica, Hospital Clínico Universidad de Chile.
}

\section{RESUMEN}

La mastitis granulomatosa idiopática es una patología inflamatoria de la mama poco frecuente, de etiología desconocida. Dado su forma de presentación clínica e imágenes puede simular tanto patología infecciosa como neoplásica, por lo que es importante sospecharla con el fin de realizar un diagnóstico adecuado y oportuno. La mayoría de los reportes en la literatura sobre esta patología corresponden a series de pocos casos. En este estudio se realizó un análisis retrospectivo de los aspectos clínicos, histopatológicos, imagenológicos y de manejo de 36 casos diagnosticados mediante biopsia Core bajo ecografía en el Hospital Clínico de la Universidad de Chile entre los años 2004 y 2014. En este reporte, al igual que en la literatura, los hallazgos clínicos e imagenológicos de la mastitis granulomatosa se superponen con los de la patología maligna e infecciosa. Si bien no observamos hallazgos patognomónicos, existen signos que hacen sospechar esta patología. En nuestro estudio encontramos que la presentación clínica más frecuente fue la masa o nódulo palpable, seguida de fístulas a la piel y engrosamiento cutáneo. Imagenológicamente el hallazgo mamográfico más frecuente fue la asimetría y en ecografía fue el área irregular con extensiones tubulares hipoecogénicas. Se revisan además aspectos histológicos, fisiopatológicos y del manejo de esta patología.

\section{PALABRAS CLAVES: Mastitis granulomatosa idiopática, mastitis, biopsia core, corticoides}

\section{SUMMARY}

Idiopathic granulomatous mastitis is an infrequent inflammatory disease of the breast, with etiology still unknown. Clinical presentation and images can simulate both infectious and neoplastic disease so it's important to suspect it to make a proper and timely diagnosis. Most reports in the literature on this topic correspond to small case series. This study is a retrospective analysis of the clinical, histopathological and imaging features and its management of 36 cases diagnosed by core biopsy under ultrasound at the Clinical Hospital of the University of Chile between 2004 and 2014. In this report, as in the literature, clinical and imaging findings in idiopathic granulomatous mastitis overlap with malignant and infectious diseases. Although we observed no pathognomonic findings, there are signs that make you suspect this condition. In our study we found that the most common clinical presentation was a palpable mass or nodule, followed by cutaneous fistula. Radiologically the most common mammographic finding was lobulated or irregular area and in ultrasound the most frecuent is the distorted breast parenchyma with posterior acoustic shadowing. Histological, pathophysiological and management aspects of this pathology was also reviewed.

\section{KEY WORDS: Idiopathic granulomatous mastitis, mastitis, core biopsy, corticoids}




\section{INTRODUCCIÓN}

La mastitis granulomatosa idiopática (MGl) es una enfermedad inflamatoria de la mama de etiología desconocida. Fue descrita por primera vez en 1972 por Kessler y Wolloch como una enfermedad que simula un cáncer inflamatorio (1). Su incidencia no ha sido determinada y en la literatura existen estudios que describen series con sólo unos pocos pacientes (2).

Se desarrolla principalmente en mujeres en edad fértil, las cuales presentan un cuadro clínico caracterizado por masa palpable, eritema, dolor, retracción del pezón y tendencia a la formación de fístulas a la piel. Puesto que puede simular otras patologías como cáncer inflamatorio, mastitis periductal, trauma e infecciones bacterianas o micóticas, su diagnóstico de certeza es un real desafío, requiriendo de confirmación histológica y de la exclusión de causas secundarias.

Imagenológicamente, ya se sea en mamografía, ecografía o resonancia magnética (RM), no existe un patrón patognomónico, pero sí algunos signos que hacen sospechar esta patología, siendo el ultrasonido el más útil de las modalidades imagenológicas (3).

El propósito de este estudio es analizar los casos de mastitis granulomatosa idiopática diagnosticados en el Centro de Imagenología del Hospital Clínico de la Universidad de Chile durante los últimos 10 años y realizar una revisión bibliográfica del tema.

\section{PACIENTES Y MÉTODOS}

Se realizó un análisis retrospectivo y descriptivo de 1681 biopsias y sus resultados histopatológicos, lo que corresponde al total de las biopsias core realizadas en el Centro de Imagenología del Hospital Clínico de la Universidad de Chile entre enero de 2004 y abril de 2014, en busca del diagnóstico de mastitis granulomatosa idiopática (MGI). De las 1681 biopsias, 51 casos demostraron MGI, 15 de los cuales fueron excluidos según los siguientes criterios: presencia de reacción de tipo cuerpo extraño en la biopsia en 9 casos, Corynebacterium (+) en 2 biopsias y Propionibacterium (+) en 1, etiología secundaria a cirugía en un caso y 1 paciente se excluyó debido a falta de datos demográficos.

Se contó, por lo tanto, con 36 casos de MGI comprobadas histológicamente, en la totalidad de los cuales se analizaron las variables de edad, sexo, lateralidad de la mama afectada, examen físico, resultado de la biopsia y hallazgos imagenológicos. El análisis de otras variables se realizó según éstas estuviesen disponibles, tales como el motivo de consulta, la duración de los síntomas, el resultado de cultivos, las tinciones histológicas y el tratamiento.

Se realizaron mamografías bilaterales análogas o digitales indirectas en un equipo Mammomat 3000 Nova marca Siemens y las ecografías se realizaron con transductor lineal de alta frecuencia 7-12 $\mathrm{MHz}$ en un equipo Philips iU22 Ultrasound System, Philips ATL 5000 o Logig 7 GE Medical System. En el caso de la paciente que posee imágenes de resonancia, éstas se realizaron en un equipo de Resonancia Magnética Superconductivo de 1,5 T con bobina dedicada e inyección de gadolinio intravenoso (Optimark) con protocolo que consistió en secuencias pre contraste potenciadas en T2 y T1 sin saturación grasa y secuencias dinámicas potenciadas en T1 Gre con fatsat. El postproceso consistió en sustracciones de las imágenes dinámicas y reconstrucciones MIP (Maximum Intensity Projection). Todas las imágenes fueron analizadas en el sistema PACS (Picture Archiving and Communication System, Agfa HealthCare).

Los datos demográficos y clínicos fueron obtenidos de las fichas clínicas, tanto las archivadas en papel como en formato electrónico y el manejo, como el uso de antibióticos, corticoides o cirugía también fueron documentados.

\section{RESULTADOS}

En el periodo estudiado un total de 36 pacientes fueron diagnosticados con mastitis granulomatosa idiopática. Todos fueron de sexo femenino, con un promedio de edad de 42,5 años (rango: 20 a 81 años).

El cuadro fue unilateral en 35 pacientes $(97 \%)$, 20 fueron derechos $(55,5 \%)$ y 15 izquierdos $(41,5 \%)$ y se presentó en forma bilateral en una paciente $(2,7 \%)$.

Los motivos de consulta fueron registrados en las fichas clínicas sólo en 28 de los 36 casos, siendo las principales causas: dolor, aumento de volumen, masa palpable, supuración espontánea, edema y retracción del pezón. A su vez la duración de los síntomas, la cual se registró en 13 casos, varió desde 3 días hasta 1 año.

La revisión de las fichas médicas en relación al examen físico demostró que la presentación individual más frecuente fue la masa o nódulo con un $55,5 \%$ de los casos, sin embargo en 10 casos se observó la presencia de más de un hallazgo en el misma mama (Tabla I).

De las 36 pacientes, sólo 18 tenían disponible mamografías en los sistemas de registro, mientras que 35 contaban con imágenes de ultrasonido al momento del análisis. Los datos de los exámenes imagenológicos se desglosan en las Tablas II y III. 
Tabla I

HALLAZGOS AL EXAMEN FÍSICO EN MUJERES CON MASTITIS GRANULOMATOSA IDIOPÁTICA

\begin{tabular}{lcc}
\hline Examen físico & $\mathrm{n}$ & $\%$ \\
\hline Nódulo o masa & 20 & 55,5 \\
Fístula cutánea - nódulo & 4 & 11,1 \\
Nódulo - engrosamiento cutáneo & 4 & 11,1 \\
Fístula cutánea & 2 & 5,5 \\
Engrosamiento cutáneo & 1 & 2,7 \\
Nódulo - engrosamiento cutáneo - fístula & 1 & 2,7 \\
Fístula cutánea - engrosamiento cutáneo & 1 & 2,7 \\
Datos no disponibles & 3 & 8,3 \\
\hline Total & 36 & 100 \\
\hline
\end{tabular}

\section{Tabla II \\ FRECUENCIAS DE HALLAZGOS MAMOGRÁFICOS EN MUJERES CON MASTITIS GRANULOMATOSA IDIOPÁTICA}

\begin{tabular}{lcc}
\hline Hallazgos mamográficos & $\mathrm{n}$ & $\%$ \\
\hline Asimetría focal & 6 & 16,6 \\
Área lobulada o irregular & 4 & 11,1 \\
Distorsión & 3 & 8,3 \\
Engrosamiento cutáneo & 2 & 5,6 \\
Normal & 3 & 8,3 \\
Sin datos & 18 & 50 \\
\hline Total & 36 & 100 \\
\hline
\end{tabular}

Tabla III

HALLAZGOS A LA ULTRASONOGRAFÍA EN MUJERES CON MASTITIS GRANULOMATOSA IDIOPÁTICA

\begin{tabular}{lcc}
\hline Hallazgos ultrasonográficos & $\mathrm{n}$ & $\%$ \\
\hline $\begin{array}{l}\text { Área irregular hipoecogénica con } \\
\text { extensiones tubulares }\end{array}$ & 14 & 38,9 \\
$\begin{array}{l}\text { Área irregular hipoecoénica sin } \\
\text { extensiones tubulares }\end{array}$ & 13 & 36,1 \\
Área - fístula & 6 & 16,7 \\
Engrosamiento cutáneo & 1 & 2,7 \\
Distorsión con sombra acústica & 1 & 2,7 \\
posterior & & \\
Fístula aislada & - & - \\
Sin datos & 1 & 2,7 \\
\hline Total & 36 & 100 \\
\hline
\end{tabular}

\section{DISCUSIÓN}

La MGI, también llamada mastitis granulomatosa globular o lobulitis granulomatosa es una patología infrecuente, de etiología desconocida que clínicamente imita otras patologías y que se presenta principalmente en mujeres en edad reproductiva. Existen en la literatura reportes de casos en niñas desde los 11 años (4), pacientes de hasta 83 años de edad (5) y presentación aislada en hombres. En nuestro estudio observamos similitudes en relación a lo descrito en la literatura con un promedio de edad de 42,5 años, con un rango de 20 a 81 años.

En la literatura no se ha descrito una clara predisposición étnica, pero sí una asociación con anticonceptivos orales y lactancia (6). Una lactancia prolongada condicionaría una distensión de los ductos y acinos lo que facilitaría la rotura de estas estructuras generando una respuesta granulomatosa (7).

A pesar de la poca información registrada en las fichas clínicas sobre la duración de la sintomatología, podemos inferir que existe una tendencia al retraso en el diagnóstico definitivo, con una duración de los síntomas que va desde los 3 días hasta 1 año, similar a lo descrito en otros reportes, posiblemente debido a lo inespecífico del cuadro y a que esta entidad imita otras patologías (3). Suelen ser pacientes que han consultado en variadas oportunidades, en las cuales se plantean varios diagnósticos diferenciales, algunas tratadas con más de un esquema antibiótico, con escasa o nula respuesta al tratamiento lo que finalmente hace plantear el diagnóstico de MGI y solicitar el estudio histológico.

La patogénesis y etiología de la MGI no está clara. Se ha descrito una teoría autoinmune mediada por células $\mathrm{T}$, que involucra primariamente un daño del epitelio ductal secundario posiblemente a trau$\mathrm{ma}$, infecciones o lactancia, lo que genera una fuga de secreciones ricas en proteínas y grasas hacia el tejido conectivo, produciendo una reacción granulomatosa, en la que participan linfocitos y macrófagos. Por otro lado esta teoría se sustenta en el hecho que muchas pacientes presentan una respuesta favorable al tratamiento con corticoides y a que se ha descrito una asociación con enfermedades de índole inmunológico, como eritema nodoso así como al aumento en el título de anticuerpos ANA en sangre (8). Otras entidades tales como sarcoidosis, granulomatosis de Wegener, mastitis de células plasmáticas, tuberculosis, brucelosis, reacción a cuerpo extraño, rotura de un quiste, ectasia ductal, necrosis grasa, infecciones parasitarias o fúngicas o específicamente por Corynebacterium sp, han sido asociadas, por lo que es imperativo descartarlas antes de plantear el diagnóstico de MGI. 
Se ha descrito una asociación entre el bacilo gram positivo Corynebacterium $\mathrm{sp}$ y el desarrollo de la MGI (9). Este germen es difícil de demostrar, debido a que es un organismo fastidioso desde el punto de vista de las necesidades que requiere para crecer en un medio de cultivo especial y a que tarda aproximadamente 72 horas en aparecer, al contrario de los cultivos corrientes que se informan a las 48 horas. Asociado a lo anterior sólo existe la posibilidad de diagnóstico por secuencias de PCR para Corynebacterium Kroppenstedtii, el tipo más frecuente desde 1998 (8). En nuestro trabajo se presentaron 2 casos positivos de Corynebacterium, diagnosticados en tinción especial al momento del análisis histológico, y ambos fueron excluidos de la base de datos final.

Histológicamente la MGI se caracteriza por una inflamación granulomatosa no caseificante compuesta por histiocitos epiteliodes, células plasmáticas, linfocitos, eosinófilos, neutrófilos, células gigantes tipo Langhans y grados variable de necrosis (Figura 1) (10). Este tipo de reacción granulomatosa se puede presentar en otras entidades como en las reacciones tipo cuerpo extraño, oleogranulomas y la tuberculosis, siendo éste último el diagnóstico diferencial histológico más importante. Para diagnosticar la mastitis tuberculosa es necesario demostrar la presencia de bacilos ácido alcohol resistentes en la tinción de Ziehl Neelsen (11), tinción que fue realizada en algunas de nuestras biopsias y sin resultados positivos.

El nódulo o masa palpable es la presentación clínica más frecuentemente descrita tanto en la literatura como en nuestra casuística (12), presentándose al examen físico, ya sea de forma aislada o simultánea con otros hallazgos al examen físico, en 29 de 36 de nuestras pacientes (80,5\%).

Otros tipos de manifestación clínica como edema, retracción del pezón, engrosamiento cutáneo, fístulas con salida a la piel y adenopatías han sido descritas, encontrando en nuestro estudio fístulas a la piel principalmente asociadas a otros hallazgos en 8 de 36 pacientes $(22,2 \%)$ y edema y engrosamiento cutáneo presentándose también acompañada de otros hallazgos en 7 pacientes $(19,4 \%)$. Son estas últimas presentaciones las que pueden sugerir un cáncer de mama y si no existe la comprobación histológica conducir erróneamente a tratamientos innecesarios como una mastectomía. En nuestro centro sólo se planteó el diagnóstico de cáncer de mama en la primera evaluación en 2 pacientes, lo cual se descartó tras el resultado histopatológico.

Existen reportes aislados de compromiso bilateral y en nuestro estudio sólo se presentó una paciente $(2,7 \%)$ con afectación bilateral comprobada histológicamente. Con respecto a la lateralidad vemos que en nuestro estudio el lado derecho fue el más frecuentemente afectado con un $55,5 \%$ de los casos (20 casos) y el izquierdo en un $41,5 \%$ ( 15 casos).

Dentro de los métodos imagenológicos utilizados para el diagnóstico de la MGI se cuenta la mamografía, el ultrasonido y la resonancia magnética. La mamografía muestra hallazgos generalmente inespecíficos como aumento difuso de la densidad, masas irregulares y asimetrías focales $(13,14,15)$, visualizando frecuentemente

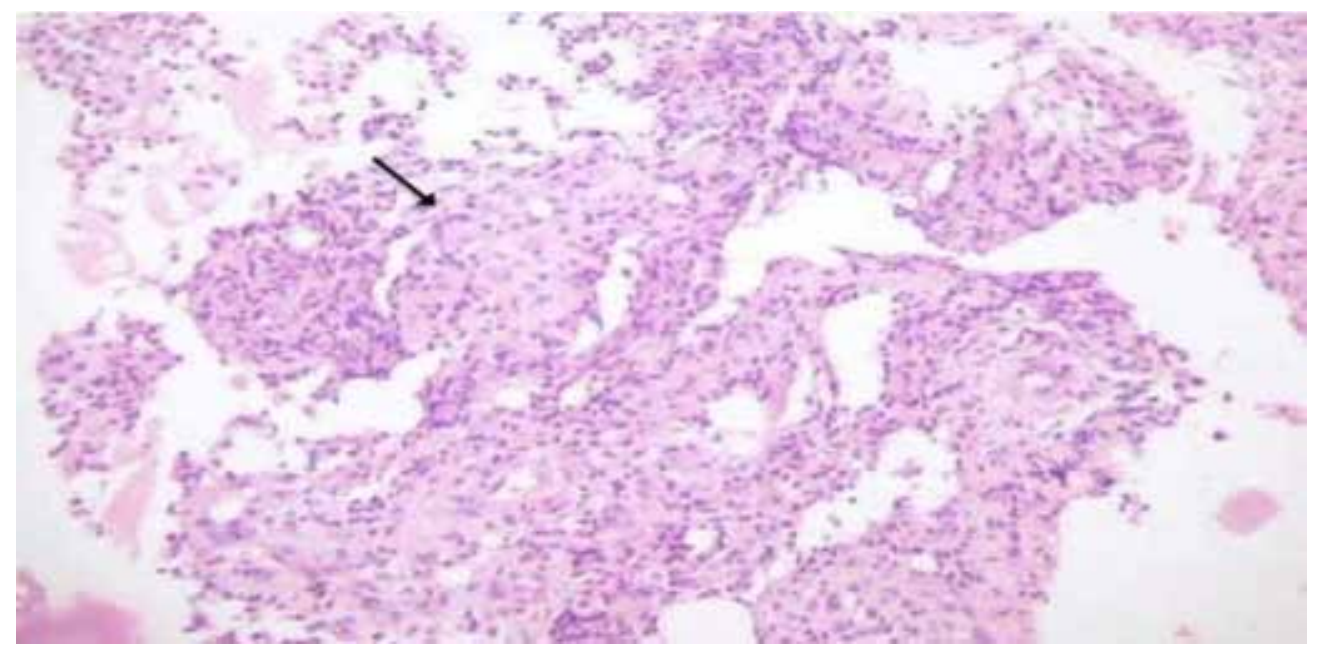

Figura 1. Corte histológico de biopsia percutánea de glándula mamaria. Foco inflamatorio rico en linfocitos e histiocitos con células gigantes multinucleadas (flecha). HE 100x. 
además engrosamiento cutáneo y retracción del pezón. Las microcalcificaciones asociadas son infrecuentes y en algunos casos la mamografía no muestra alteraciones. En nuestro estudio sólo 18 de las 36 pacientes tenían disponibles sus mamografías, y de este total se encontró que el hallazgo más frecuente fue la asimetría focal en el 16,6\% (6 casos) seguido de un área lobulada o irregular en el 11,1\% (4 casos) (Figuras 2 y 3 ). La distorsión y el engrosamiento cutáneo como hallazgo aislado lo encontramos en el 8,3\% (3 casos) y $5,6 \%$ ( 2 casos) respectivamente, en 3 casos $(8,3 \%)$ las mamografías no presentaron hallazgos.

El ultrasonido es una buena herramienta de aproximación diagnóstica en el análisis de la MGI (13). Se han descrito lesiones irregulares, anfractuosas, hipoecogénicas, heterogéneas, mal definidas y múltiples, que tienden a coalescer y que pueden presentar refuerzo o sombra acústica posterior, asociado a tractos sinuosos hipoecogénicos que son extensiones tubulares hipoecogénicas a través de los lóbulos. Se ha descrito también la presencia de adenopatías axilares y edema circundante $(13,14,15)$.

En 35 de 36 de nuestras pacientes se obtuvo las imágenes ecográficas al momento del análisis, encontrándose que el hallazgo más frecuente fue un área irregular hipoecogénica con extensiones tubulares en 14 pacientes (38,9\%) seguido por un

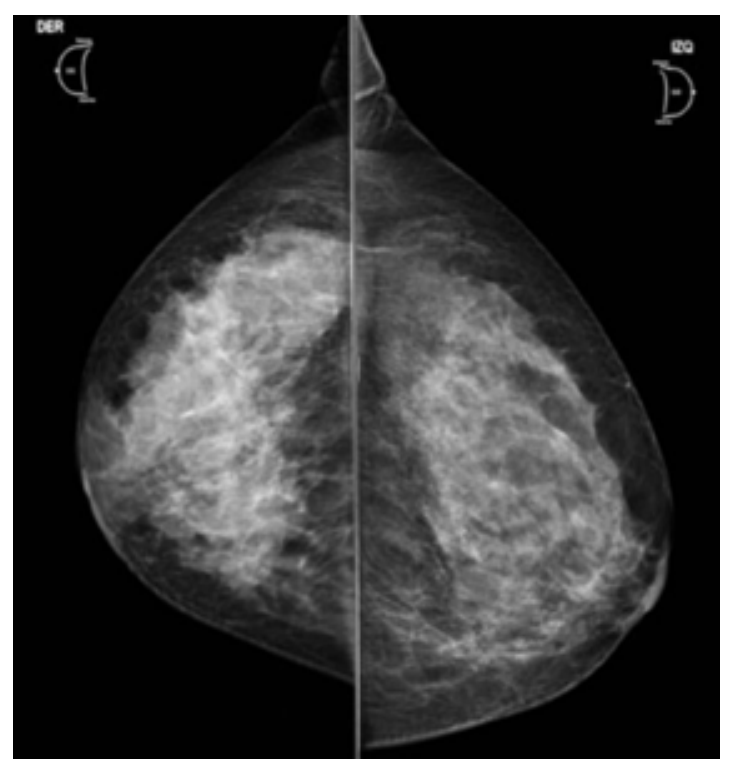

Figura 2. Mujer de 42 años. Mamografía proyección cráneo caudal. Mamas heterogéneamente densas con aumento de densidad global de bordes mal definidos de la mama derecha.

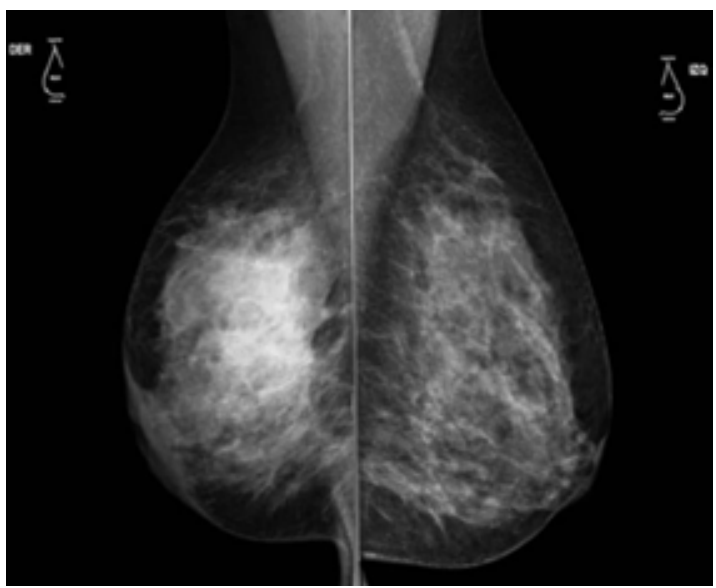

Figura 3. Mujer 42 años. Mamografía proyección mediolateral oblicua. Mamas heterogéneamente densas con aumento de densidad global de mama derecha.

área irregular hipoecogénica sin extensiones tubulares en 13 casos $(36,1 \%)$ y de un área hipoecogénica irregular asociada a una fístula cutánea en 6 pacientes (16,7\%) (Figuras 4, 5, y 6). El engrosamiento cutáneo aislado, no asociado a otros hallazgos sólo se vio en 1 caso $(2,7 \%)$. Es relevante mencionar que si bien la presencia de fístulas no fue el hallazgo más frecuente es un signo altamente sugerente de esta patología, especialmente cuando son recurrentes y es de utilidad al momento de diferenciarla de otros tipos de mastitis.

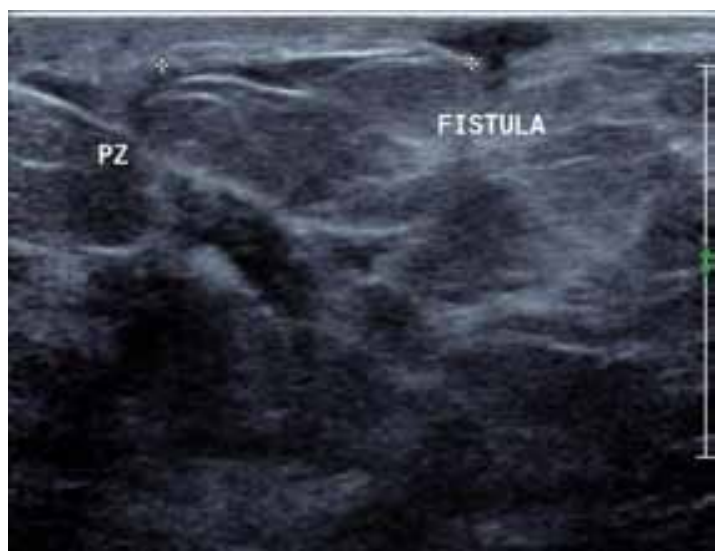

Figura 4. Mujer 31 años, aumento de volumen, eritema de 4 meses evolución y fístulas a piel. En ultrasonido se observa aumento de la ecogenicidad de la piel y del subcutáneo con engrosamiento del plano cutáneo más evidente en cuadrantes superiores e internos izquierdos asociado a múltiples fístulas con y sin salida a la piel. 


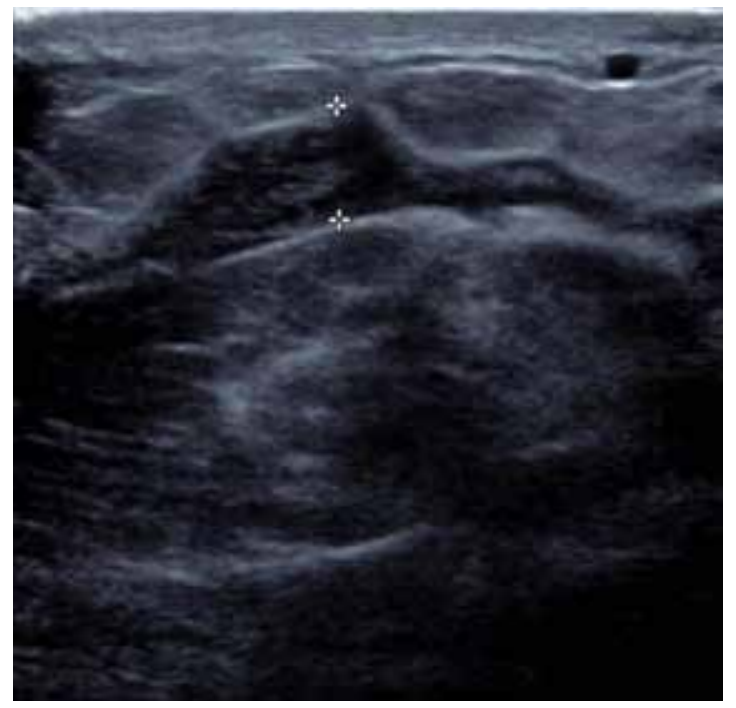

Figura 5. Mujer del caso 3. Se observa una colección que se dirige desde la región retroareolar hacia el cuadrante súpero interno, hora 9 - 10 con una longitud estimada de $8 \mathrm{~cm}$.

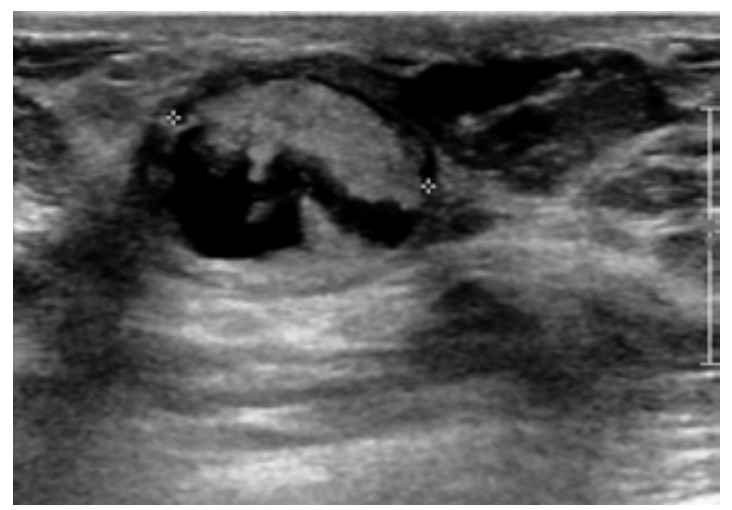

Figura 6. Mujer 68 años, con eritema, dolor y retracción del pezón. En ultrasonido se visualiza un nódulo sólido quístico retroareolar derecho, no vascularizado.

En nuestro estudio una paciente se realizó RM contrastada evidenciando en la mama alterada, áreas difusas de mayor intensidad de señal en secuencias potenciadas en T2 y en secuencias con gadolinio zonas de realce en anillo no mayores a $10 \mathrm{~mm}$ (Figuras 7 y 8 ).

En la literatura se describe la presentación de un patrón de realce con áreas regionales o segmentarias de tipo no masa, asociada a lesiones en anillo, que pueden ser de milímetros a centímetros y que reflejan microabscesos asociados al proceso granulomatoso inflamatorio en el tejido mamario. Por otra parte las lesiones son hiperintensas en secuencias potenciadas en T2, y en las potenciadas en $T 1$, la señal muestra variabilidad dependiendo del contenido proteico de las lesiones. Cuando se administra gadolinio y se analizan las curvas dinámicas se visualizan lesiones en anillo con una fase precoz de ascenso rápido, y una fase postinicial y tardía tipo lavado, asociadas a áreas de realce tipo no masa con una curva ascendente $(2,16)$. La RM es válida para evaluar la extensión del proceso inflamatorio y es una herramienta útil para realizar el seguimiento post tratamiento con el fin de evaluar la presencia de enfermedad residual.

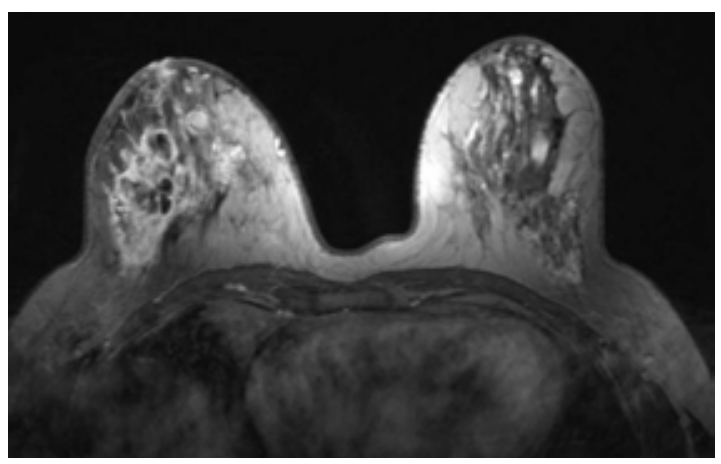

Figura 7. Adquisición axial en secuencia potenciada en T2. Mujer de 42 años. En la mama derecha se observa área de mayor intensidad de señal que compromete prácticamente toda la mama, de comportamiento heterogéneo, con marcado realce cutáneo, en especial en situación periareolar.

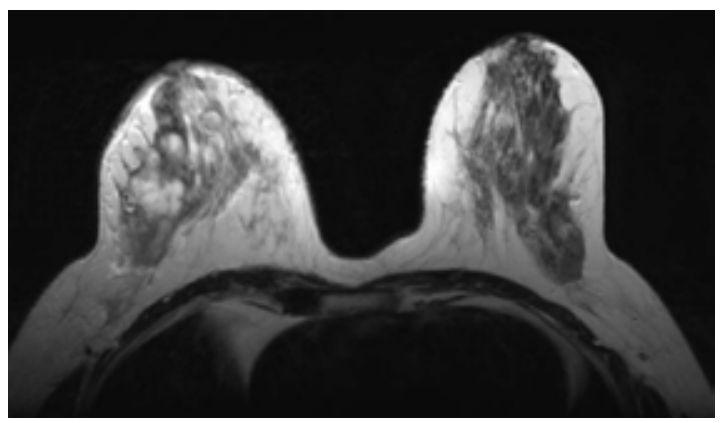

Figura 8. Adquisición axial en secuencia dinámica con gadolinio, minuto 2 . En la mama derecha se identifican algunas áreas de impregnación de tipo progresiva de realce en anillo no mayores de 10 $\mathrm{mm}$ de distribución dispersa mayoritariamente en cuadrantes externos. 
El manejo óptimo de la MGI es controversial. Se describen variados tratamientos, desde el uso de corticoides y antibióticos como terapia conservadora y escisión quirúrgica ya sea parcial o total. En pacientes con mastitis no complicada podría mantenerse una conducta expectante sobre la base de casos reportados con resolución espontánea (17). Los corticoides han sido empleados para el tratamiento tanto curativo como con el objetivo de reducir el proceso inflamatorio para posteriormente realizar una extirpación quirúrgica, incluso con reconstrucción estética inmediata $(18,19)$. El tratamiento quirúrgico descrito en la literatura incluye escisión de la masa o nódulo y drenaje de las colecciones, manejo que sin duda depende de cada paciente (6). Un punto importante a destacar es que la terapia antibiótica no tiene sustento etiológico para su uso en esta patología, que por definición no presenta gérmenes asociados por lo que su prescripción no estaría justificada.

En nuestro estudio identificamos 14 pacientes con su respectivo manejo, el cual se basó principalmente en corticoides asociados a antiinflamatorios no esteroidales y/o antibióticos, y en 2 casos se requirió cirugía, realizándose una mastectomía parcial y total debido a recurrencias y numerosos fracasos de tratamientos farmacológicos.

\section{CONCLUSIÓN}

La MGI es una enfermedad infrecuente de etiología poco clara. Ocurre predominantemente en mujeres premenopaúsicas y por su presentación clínica inespecífica suele ser confundida con enfermedad inflamatoria y/o neoplásica. No hay características patognomónicas, sin embargo suelen existir signos clínicos e imagenológicos que permiten sospechar esta patología, evitando un retraso en el diagnóstico. Dentro de ello, destaca la ecografía como estudio de mayor rendimiento, por los hallazgos de áreas hipoecogénicas, anfractuosas, conectadas entre sí, con extensiones tubulares a la piel, como la manifestación más frecuente y sugerente de MGI. Permitiendo además la realización de biopsia core para confirmación diagnóstica (gold standard). El manejo continúa siendo controversial.

\section{REFERENCIAS}

1. Kessler E, Wolloch Y. Granulomatous mastitis: a lesion clinically simulating carcinoma. Am J Clin Pathol 1972;58:642-6.
2. Hovanessian L, Peyvandi B, Klipfel N, Grant E, lyengar $\mathrm{G}$. Granulomatous lobular mastitis: imaging, diagnosis, and treatment. AJR 2009; 193:574-81.

3. Gunduz Y, Altintoprak F, Tatli Ayhan L, Kivilcim T, Celebi F. Effect of Topical Steroid Treatment on Idiopathic Granulomatous mastitis: clinical and radiologic evaluation. Breast J 2014;20:586-91.

4. Bani-Hani KE, Yaghan RJ, Matalka II, Shatnawi NJ. Idiopathic granulomatous mastitis: time to avoid unnecessary mastectomies. Breast J 2004;10:31822.

5. Lai EC, Chan WC, Ma TK, Tang AP, Poon CS, Leong HT. The role of conservative treatment in idiopathic granulomatous mastitis. Breast J 2005;11:454-6.

6. Kok KY, Telisinghe PU. Granulomatous mastitis: Presentation, treatment and outcome in 43 patients. Surgeon 2010;8:197-201.

7. Kaur AC, Dal H, Muezzinoglu B, Paksoy N. Idiopathic granulomatous mastitis. Report of a case diagnosed with fine needle aspiration cytology. Acta Cytol 1999;43:481-4.

8. Ozel L, Unal A, Unal E, Kara M, Erdoğdu E, Krand $O$, et al. Granulomatous mastitis: is it an autoimmune disease? Diagnostic and therapeutic dilemmas. Surg Today 2012;42:729-33.

9. Bercot B, Kannengiesser C, Oudin C, Grandchamp B, Sanson-le Pors MJ, Mouly S, Elbim C. First description of NOD2 variant associated with defective neutrophil responses in a woman with granulomatous mastitis related to corynebacteria. J Clin Microbiol 2009;47:3034-7.

10. Taylor GB, Paviour SD, Musaad S, Jones WO, Holland DJ. A clinicopathological review of 34 cases of inflammatory breast disease showing an association between corynebacteria infection and granulomatous mastitis. Pathology 2003; 35:109-19.

11. Lacambra M, Thai TA, Lam CC, Yu AM, Pham HT, Tran PV, et al. Granulomatous mastitis: the histological differentials. J Clin Pathol 2011;64:405-11.

12. Hee Ri Na Seo, Kuk Young Na, Hyun Ee Yim, Tae Hee Kim, Doo Kyoung Kang, Ki Keun Oh, et al. Differential diagnosis in idiopathic granulomatous mastitis and tuberculous mastitis. J Breast Cancer 2012;15:111-8.

13. Erhan Y, Veral A, Kara E, Özdemir N, Kapkac M, Özdedeli E, et al. A clinicopathologic study of a rare clinical entity mimicking breastcarcinoma: idiopathic granulomatous mastitis. Breast 2000;9:52-6.

14. Gautier N, Lalonde L, Tran-Thanh D, El Khoury M, David J, ,Labelle Maude, et al. Chronic granulomatous mastitis: Imaging, pathology and management. Eur J Radiology 2013;82:165-75.

15. Boufettal H, Essodegui F, Noun M, Hermas S, Samouh N. Idiopathic granulomatous mastitis: A report of twenty cases. Diagn Interv Imaging 2012;93:586-96.

16. Al-Khawari HA, Al-Manfouhi HA, Madda JP, Kovacs A, Sheikh M, Roberts O. Radiologic features of granulomatous mastitis. Breast J 2011;17:645-50.

17. Lai EC, Chan WC, Ma TK, Tang AP, Poon Cs Leong 
$\mathrm{HT}$. The role of conservative treatment in idiopathic granulomatous mastitis. Breast J 2005;11:454-6.

18. Sakurai K, Fujisaki S, Enomoto K, Amano S, Sugitani M. Evaluation of follow-up strategies for corticosteroid therapy of idiopathic granulomatous mastitis. Surg Today 2011;41:333-7.
19. Hladik M, Schoeller $T$, Ensat $F$, Wechselberger G. Idiopathic granulomatous mastitis: Successful treatment by mastectomy and immediate breast reconstruction. J Plast Reconstr Aesthet Surg 2011;64:1604-7. 